\title{
Méthodes probabilistes et déterministes d'estimation des débits extrêmes : comparaison de résultats sur deux bassins versants de Colombie-Britannique (Canada)
}

\author{
Pierre BRIGODE ${ }^{1,2}$, Zoran MIĆOVIĆ ${ }^{3}$, Pietro BERNARDARA ${ }^{1}$, Emmanuel PAQUET ${ }^{4}$, Joël GAILHARD ${ }^{4}$, \\ Federico GARAVAGLIA ${ }^{4}$, Rémy GARÇON ${ }^{4}$ et Pierre RIBSTEIN ${ }^{2}$ \\ 1. LNHE, R\&D, Electricité de France, Chatou, France \\ 2. UMR 7619 Sisyphe, Université Pierre et Marie Curie, Paris, France. Actuellement École de Technologie Supérieure et Ouranos, Montréal, \\ Québec, Canada. brigode.pierre@ouranos.ca \\ 3. BC Hydro, Engineering, Burnaby, British Columbia, Canada \\ 4. DTG, DMM, Électricité de France, Grenoble, France
}

\begin{abstract}
RÉSUMÉ. - Deux méthodes d'estimation des débits extrêmes ont été appliquées sur deux bassins versants situés sur la côte Pacifique de la Colombie-Britannique, province canadienne : la méthode déterministe classique Probable Maximum Precipitation (PMP) / Probable Maximum Flood (PMF) et la méthode probabiliste Simulation Climato-Hydrologique pour l'Appréciation des Débits EXtrêmes (SCHADEX). Les ratios entre les valeurs de PMP et les pluies journalières de période de retour 10000 ans estimées par la méthode SCHADEX sont proches de 1, alors que les ratios entre les valeurs de PMF et les débits journaliers de période de retour 10000 ans estimés par la méthode SCHADEX sont supérieurs à 1.25. Ces valeurs de ratios d'estimation de débits extrêmes sont du même ordre de grandeur que celles obtenues lors de la comparaison de valeurs de PMF et d'estimations de débits extrêmes réalisées avec la méthode du GRADEX [Guillot et Duband 1967] pour 43 bassins versants, pour lesquels les valeurs de ratios étaient comprises entre 1.08 et 3.52 [CFGB, 1994]. Cette communication illustre la nécessité de réaliser des estimations de pluies et crues extrêmes avec les mêmes entrées hydroclimatiques (et notamment des séries de pluies de bassin construites avec les mêmes hypothèses) pour réaliser une comparaison rigoureuse de méthodes de prédétermination. Une telle approche permettrait notamment de discuter des différences de transformation pluie-débit réalisées par les différents modèles hydrologiques considérés.
\end{abstract}

Mots-clés : Prédétermination des débits extrêmes, PMP, PMF, MEWP, SCHADEX.

\section{Probabilistic and deterministic extreme flood estimation methods: Estimation comparison on two British Columbia catchments (Canada)}

\begin{abstract}
Two different extreme streamflow estimation methods have been applied and compared over two catchments located on the Pacific coast of the British Columbia Canadian province: the classical deterministic approach Probable Maximum Precipitation (PMP) / Probable Maximum Flood (PMF) and the Simulation Climato-Hydrologique pour l'Appréciation des Débits EXtrêmes (SCHADEX) probabilistic approach, aiming at stochastically simulating millions of floods. Ratios between PMP values and 10000-year return period daily precipitation values estimated by the SCHADEX method are close to 1, while ratios between PMF values and 10000-year return period daily streamflow values estimated by the SCHADEX method are greater than 1.25. These ratio values are similar to the ones obtained within the comparison of PMF values and extreme streamflow estimation performed with the GRADEX method [Guillot and Duband 1967] for 43 different catchments, with ratios ranging between 1.08 and 3.52 [CFGB, 1994]. This paper illustrates the need of estimating extreme rainfall and streamflow by using the same hydroclimatologic inputs (in particular for the catchment rainfall series which should be estimated with the same catchment water balance hypothesis) in order to properly compare extreme rainfall and flood estimation methods. Such an approach allows to discuss the rainfallrunoff transformation differences obtained when different hydrological models are considered.
\end{abstract}

Key-words: Estimation of extreme streamflow, PMP, PMF, MEWP, SCHADEX.

\section{INTRODUCTION}

Électricité de France (EDF) et BC Hydro (BCH) sont deux des leaders de la production hydroélectrique en Europe et en Amérique du Nord. Leurs programmes de recherche sur la sûreté des ouvrages permettent une veille constante sur les méthodes d'estimation de l'aléa hydrologique, afin d'assurer la sûreté de leurs installations. EDF a récemment développé une nouvelle méthode d'estimation des débits extrêmes, la méthode Simulation Climato-Hydrologique pour l'Appréciation des Débits Extrêmes (SCHADEX), qui est utilisée depuis 2006 pour dimensionner les évacuateurs de crues de ses barrages [Paquet et al. 2006, 2013, Garavaglia 2011]. Afin de partager leur expérience en termes de comparaison des débits extrêmes, une collaboration scientifique entre EDF et BCH a été entamée en 2011. L'objectif principal de cette collaboration a été l'application de la méthode SCHADEX sur deux bassins versants de Colombie-Britannique : la rivière Campbell au barrage de Strathcona (bassin versant pluvio-nival de $1193 \mathrm{~km}^{2}$, noté 
bassin de la Campbell par la suite) et la rivière Coquitlam au barrage de Coquitlam (bassin versant pluvio-nival de $188 \mathrm{~km}^{2}$, noté bassin de la Coquitlam par la suite). Par ailleurs, $\mathrm{BCH}$ a testé et appliqué plusieurs méthodes d'estimation des débits extrêmes sur les bassins versants où sont construits ses barrages, avec notamment la méthode déterministe classique Probable Maximum Precipitation (PMP) / Probable Maximum Flood (PMF) et plus récemment la méthode probabiliste de simulation stochastique de crues Stochastic Event Flood Model (SEFM), appliquée sur certains bassins versants [Schaefer et Barker, 2009].

Peu de comparaisons entre estimations déterministes et estimations probabilistes existent dans la littérature. Parmi celles existantes, le Comité Français des Grands Barrages (CFGB) a comparé les estimations de débits de période de retour 10000 ans réalisées avec la méthode du GRADEX [Guillot et Duband 1967] et les valeurs de PMF sur 43 bassins versants différents (dont 19 bassins versants français), montrant que les valeurs de PMF étaient entre 1.08 et 3.52 fois plus importantes que les débits estimés par GRADEX pour des périodes de retour de 10000 ans, avec une valeur moyenne de 2.07 [CFGB, 1994].

Cette communication vise à présenter les résultats de l'application de la méthode SCHADEX sur deux bassins versants situés dans la région côtière de Colombie-Britannique : le bassin de la Campbell et celui de la Coquitlam. Les estimations de débits extrêmes obtenues par la méthode SCHADEX seront comparées aux estimations d'ores et déjà réalisées par $\mathrm{BCH}$ grâce à la méthode $\mathrm{PMP} / \mathrm{PMF}$.

\section{MÉTHODES D'ESTIMATION DE DEBITS EXTRÊMES}

Deux méthodes d'estimation de débits extrêmes ont été appliquées, puis comparées sur deux bassins versants Canadiens : la méthode déterministe PMP/PMF et la méthode probabiliste SCHADEX. Les méthodes déterministes se proposent d'estimer le débit de crue maximal pouvant être observé sur le bassin versant étudié, en combinant de manière défavorable - mais crédible - des situations hydrométéorologiques observées ou extrapolées à leur maximum supposé. Les méthodes probabilistes visent, quant à elles, à définir la distribution de probabilité des crues extrêmes sur le bassin versant étudié et donc à associer une période de retour aux valeurs de débits extrêmes.

\section{II.1. Probable Maximum Precipitation (PMP) et Probable Maximum Flood (PMF)}

$\mathrm{BCH}$ a appliqué la méthode d'estimation de PMP développée par le Service National Météorologique des États-Unis [Hansen 1987]. Une expertise hydrométéorologique importante permet ainsi d'estimer la PMP du bassin versant étudié à partir de l'analyse d'épisodes historiques majeurs, en maximisant de nombreux paramètres, tels que la durée de l'épisode pluvieux, la quantité d'eau précipitée, la distribution spatiale des précipitations, etc. Une analyse détaillée de ces variables, de leurs interactions et de leurs contributions respectives à l'incertitude liée à l'estimation de la PMP est détaillée par Mićović et al. [2012].

Deux valeurs de PMF ont ensuite été estimées, selon les recommandations de l'Association Canadienne des Barrages [CDA 2007], en combinant notamment des situations défavorables de précipitation et d'accumulation de neige :

1. La PMF automnale ou estivale, générée par la PMP ;

2. La PMF printanière, définie comme étant le maximum de deux cas :

a. PMF générée par la combinaison d'une PMP printanière et d'une accumulation de neige de période de retour 100 ans.

b. PMF générée par un épisode pluvieux de période de retour 100 ans et l'accumulation de neige maximale probable.

D'autres scénarios ont également été testés par BCH et sont détaillés dans le Tableau 1. Ces différentes combinaisons défavorables de précipitation et de neige sont transformées en débit grâce au modèle pluie-débit UBC Watershed Model [Quick 1995 ; Mićović et Quick 1999], préalablement calé sur les bassins versants étudiés.

\section{II.2. Simulation Climato-Hydrologiques pour l'Appréciation des Débits Extrêmes (SCHADEX)}

Depuis 2006, EDF applique une nouvelle approche climato-hydrologique de prédétermination des pluies et crues extrêmes, la méthode SCHADEX, pour le dimensionnement des évacuateurs de crues [Paquet et al. 2006, 2013, Garavaglia 2011]. Cette méthode intègre un processus de simulation dite "semi-continue » visant à croiser exhaustivement les aléas pluie extrême et état hydrologique du bassin considéré (i.e. simuler la réponse hydrologique de pluies de tous ordres tombant sur tous les états hydrologiques possibles du bassin). SCHADEX couple pour cela deux modèles : (i) un modèle probabiliste de pluie (basé sur

Tableau 1 : Exemples de scénarios de PMF testés par BCH sur un bassin versant de Colombie Britannique, pour lequel le scénario produisant la plus forte valeur de PMF est le scénario CDA 2 testé en février. L'abréviation $\mathrm{V}_{100 \text { ans }}$ signifie valeur de période de retour 100 ans.

\begin{tabular}{|l|l|l|l|l|l|}
\hline Nom du scénario & \multicolumn{1}{|c|}{ Mois } & \multicolumn{1}{|c|}{$\begin{array}{c}\text { Accumulation } \\
\text { de neige }\end{array}$} & $\begin{array}{c}\text { Conditions } \\
\text { de températures }\end{array}$ & $\begin{array}{c}\text { Pluies précèdent } \\
\text { l'épisode pluvieux }\end{array}$ & Episode pluvieux \\
\hline CDA 1 & Avril & Maximale & Tempête & Aucune & $\mathrm{V}_{100 \text { ans }}$ \\
\hline CDA 2 & Avril & $\mathrm{V}_{100 \text { ans }}$ & Tempête & Aucune & PMP \\
\hline CDA 2 & Novembre & $\mathrm{V}_{100 \text { ans }}$ & Tempête & Aucune & PMP \\
\hline CDA 2 & Février & $\mathrm{V}_{100 \text { ans }}$ & Tempête & Aucune & PMP \\
\hline CDA 3 & Avril & Maximale & « Fonte critique $»$ & Aucune & Aucun \\
\hline BCH 1 & Avril & Moyenne & Tempête & $\mathrm{V}_{100 \text { ans }}$ & PMP \\
\hline BCH 2 & Février & Moyenne & Tempête & $\mathrm{V}_{100 \text { ans }}$ & PMP \\
\hline
\end{tabular}


un sous-échantillonnage par type de temps et par saison) décrivant l'aléa pluie extrême du bassin versant étudié et (ii) un modèle hydrologique décrivant les processus de transformation pluie-débit à l'échelle du bassin. Le modèle probabiliste de pluie le plus couramment utilisé est le modèle Multi-Exponential Weather Patterns (MEWP), décrit par Garavaglia et al. [2010, 2011] et le modèle hydrologique utilisé est le modèle MORDOR, décrit par Garçon [1999].

\section{DEUX BASSINS VERSANTS DE LA CÔTE PACIFIQUE DU CANADA}

\section{III.1. Contexte hydro-climatique}

Les deux bassins versants étudiés se trouvent sur la région côtière de Colombie-Britannique : la rivière Campbell s'écoule sur la partie intérieure de l'île de Vancouver alors que la rivière Coquitlam s'écoule sur le continent, à quelques kilomètres au nord-est de Vancouver. Ces bassins versants pluvio-nivaux sont de taille moyenne (respectivement $1193 \mathrm{~km}^{2}$ et $188 \mathrm{~km}^{2}$ ) et sont caractérisés par des gammes d'altitude assez larges (altitudes respectivement comprises entre 134 et $2192 \mathrm{~m}$ et entre 153 et $1173 \mathrm{~m}$ ). La Figure 1 présente la localisation de ces deux bassins versants, ainsi que leur topographie et leur régime hydro-climatique. Si la saisonnalité des régimes est similaire pour les deux bassins versants, l'amplitude des cumuls est différente : pour le bassin de la Campbell, la pluie annuelle moyenne est de $2440 \mathrm{~mm}$ pour un écoulement annuel moyen de $2036 \mathrm{~mm}$; alors que pour le bassin de la Coquitlam, la pluie annuelle moyenne est de $4212 \mathrm{~mm}$, pour un écoulement annuel moyen de $3570 \mathrm{~mm}$. Ces différences de cumuls s'expliquent principalement par les différences de position et d'orientation des deux bassins versants. Le bassin de la Campbell se trouve en effet sur la face est de l'île de Vancouver et est donc relativement « protégé » des flux originaires de l'océan Pacifique, contrairement au bassin de la Coquitlam, qui est plus directement exposé à ces flux océaniques.

Les deux bassins versants sont soumis à des épisodes pluvieux intenses d'octobre à mars, saisonnalité généralisable à l'ensemble de la région côtière de Colombie-Britannique. Une classification en cinq types de temps a été établie sur cette région de Colombie-Britannique afin de discriminer l'origine synoptique des épisodes pluvieux observés sur cette région et d'identifier les situations typiques et récurrentes [Brigode et al. 2013]. Parmi ces cinq types de temps, un type de temps particulier (le type de temps 3, qui regroupe
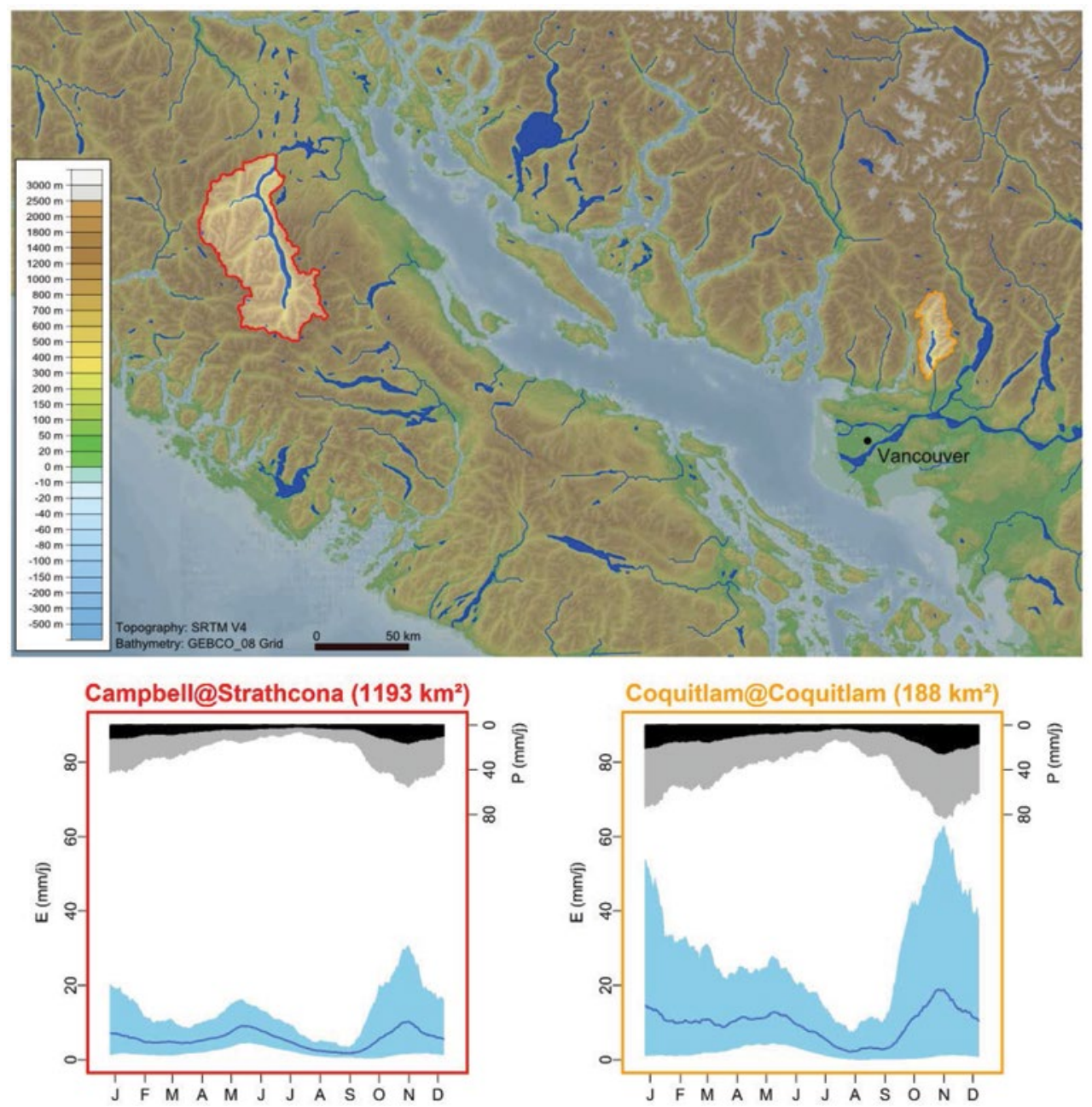

Figure 1 : Localisation, topographie et régime hydro-climatique des deux bassins versants étudiés (établis sur la période 1981-2010) : le bassin de la Campbell en rouge et le bassin de la Coquitlam en orange. Les régimes ont été estimés sur des fenêtres glissantes de 20 jours : les lignes bleues représentent les écoulements journaliers moyens, les enveloppes bleu-ciel sont construites avec les quantiles $5 \%$ et $95 \%$ des écoulements journaliers, les enveloppes noires représentent les pluies journalières moyennes et les enveloppes grises représentent les quantiles $95 \%$ des pluies journalières. 
des journées caractérisées par des circulations du sud-ouest vers le nord-est, particulièrement pluvieuses dans la partie centrale de l'île de Vancouver) s'est révélé être à l'origine de la majeure partie des épisodes pluvieux intenses sur le bassin de la Campbell. Contrairement au bassin de la Campbell, plusieurs types de temps (notamment les types de temps 2, 3 et 4) peuvent être à l'origine d'épisodes pluvieux intenses sur le bassin de la Coquitlam.

Ces deux bassins versants sont également influencés par des phénomènes nivaux, avec des volumes significatifs de neige accumulés pendant l'hiver, générant ensuite une onde de fonte durant les mois de mai et juin. Les crues les plus intenses sont observées sur les deux bassins versants durant le mois de novembre et sont souvent produites par des épisodes pluvieux particulièrement intenses, parfois aggravés par des phénomènes de fusion du manteau neigeux précoce.

\section{III.2. Données utilisées pour l'application de la méthode SCHADEX}

L'ensemble des chroniques hydrométéorologiques disponibles sur les deux bassins versants étudiés a été utilisé pour l'application de la méthode SCHADEX. Ainsi, plusieurs séries de pluies et de températures journalières ont été utilisées sur chaque bassin versant : trois stations avec 30 années de données (de 1981 à 2010) pour le bassin de la Campbell et deux stations avec 50 années de données (de 1960 à 2010) pour le bassin de la Coquitlam. Ces différentes séries de pluies ponctuelles ont été combinées linéairement pour constituer une seule série de pluies de bassin pour chaque bassin versant, considérée ensuite comme représentative de la totalité des précipitations reçues par le bassin versant. Ces séries de pluies de bassin ont donc été utilisées en entrée du modèle hydrologique MORDOR et ont également été utilisées pour étudier les propriétés statistiques des épisodes pluvieux observés sur chacun des deux bassins versants. 51 années de débits journaliers (de 1960 à 2010)
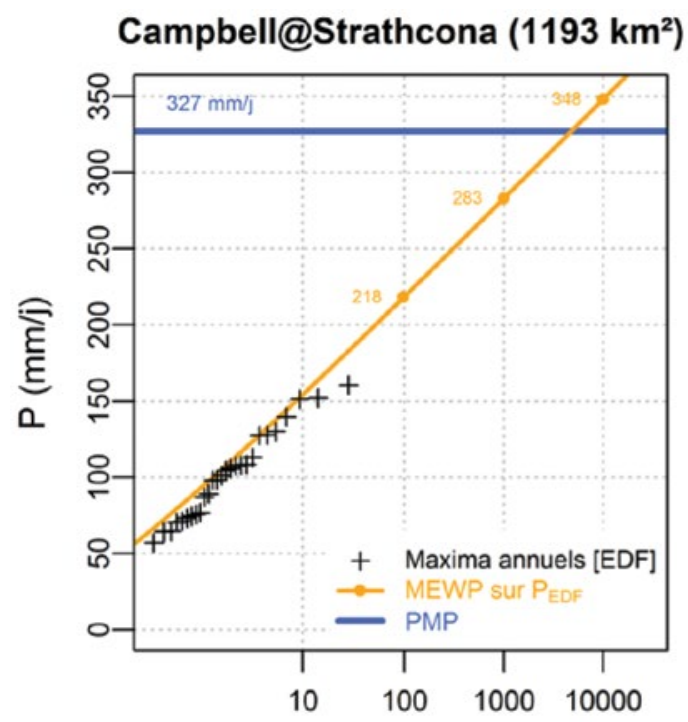

Temps de retour (années) sont également disponibles pour chacun des deux bassins versants. Ces séries de débits ont notamment été utilisées pour caler le modèle hydrologique MORDOR.

Une série de pluies journalières de bassin définie par $\mathrm{BCH}$ étant disponible pour le bassin de la Coquitlam, une estimation de débits extrêmes par la méthode SCHADEX a été réalisée avec cette série de pluie de bassin (définition du modèle probabiliste MEWP, de l'aléa de saturation du bassin versant et calage du modèle hydrologique MORDOR avec cette série), puis comparée avec l'estimation de débits extrêmes réalisée en considérant la pluie de bassin construite par EDF.

\section{RÉSULTATS}

\section{IV.1. Estimations de pluies extrêmes}

La Figure 2 présente, pour les deux bassins versants étudiés, les distributions des pluies journalières estimées par le modèle MEWP de la méthode SCHADEX, comparées avec des distributions de maxima annuels de pluies journalières et les valeurs de PMP estimées par $\mathrm{BCH}$.

Les valeurs de PMP estimées par BCH sont comprises entre les pluies de période de retour 1000 ans et 10000 ans pour le bassin de la Campbell (environ 4800 ans) et supérieure à la pluie de période de retour 10000 ans pour le bassin de la Coquitlam (environ 45000 ans lorsque le modèle MEWP utilisant la pluie de bassin EDF est considéré). Des ratios entre les valeurs de PMP estimées et les valeurs de pluies journalières de période de retour 10000 ans estimées par le modèle MEWP ont été calculés pour chaque bassin versant et sont reportés dans la partie gauche du Tableau 2. Les valeurs de ratios obtenues semblent être faibles, notamment pour le bassin de la Campbell (ratio de 0.94) : les valeurs de PMP sont en effet classiquement associées à des temps de retour supérieurs à 10000 ans.

\section{Coquitlam@Coquitlam (188 km²)}

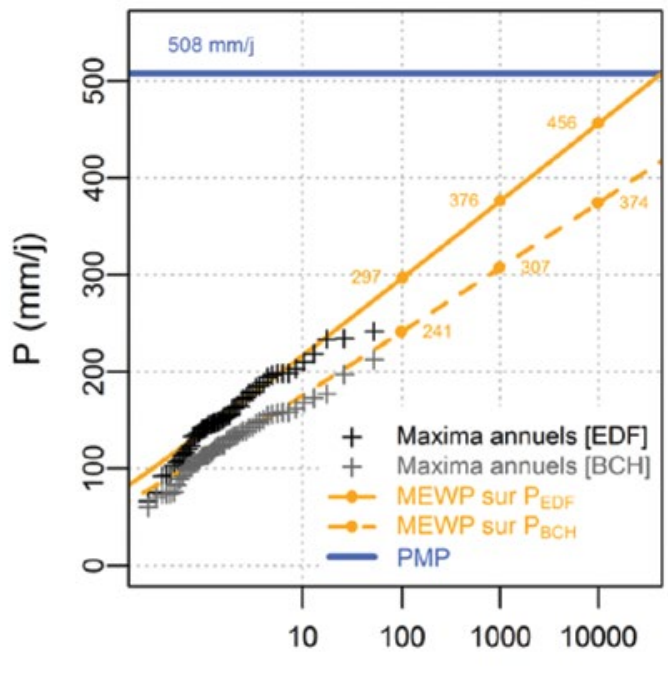

Temps de retour (années)

Figure 2 : Bassins versants de la Campbell (à gauche) et de la Coquitlam (à droite) : distributions des pluies journalières estimées par le modèle MEWP utilisant la pluie de bassin EDF (ligne orange) et par le modèle MEWP utilisant la pluie de bassin $B C H$ (tirets oranges), comparées aux maxima annuels obtenus avec les pluies de bassin construites par EDF (croix noires), aux maxima annuels obtenus avec les pluies de bassin construites par BCH (croix grises) et aux estimations de PMP réalisées par BCH (ligne bleue). 
Tableau 2 : Estimations SCHADEX de pluie journalière (partie gauche) et de débit journalier (partie droite) de période de retour 10000 ans, comparées avec des valeurs de PMP et de PMF, pour les bassins de la Campbell et de la Coquitlam.

\begin{tabular}{|c|c|c|c|c|c|}
\hline & Campbell & Coquitlam & & Campbell & Coquitlam \\
\hline $\mathbf{P}_{1000 \text { EDF }}(\mathbf{m m} / \mathbf{j})$ & 348 & 456 & $\mathbf{Q}_{10000 \mathrm{EDF}}\left(\mathbf{m}^{\mathbf{3}} / \mathbf{s}\right)$ & 3209 & 842 \\
\hline $\mathbf{P}_{10000 \mathrm{BCH}}(\mathbf{m m} / \mathbf{j})$ & - & 374 & $\mathbf{Q}_{10000 \mathrm{BCH}}\left(\mathbf{m}^{\mathbf{3}} / \mathbf{s}\right)$ & - & 780 \\
\hline $\mathbf{P M P}(\mathbf{m m} / \mathbf{j})$ & 327 & 508 & $\mathbf{P M F}\left(\mathbf{m}^{\mathbf{3}} / \mathbf{s}\right)$ & 4450 & 1074 \\
\hline $\mathbf{P M P} / \mathbf{P}_{\mathbf{1 0 0 0 0 \mathrm { EDF }}}[-]$ & 0.94 & 1.11 & $\mathbf{P M F} / \mathbf{Q}_{\mathbf{1 0 0 0 0 \mathrm { EDF }}}[-]$ & 1.39 & 1.28 \\
\hline $\mathbf{P M P} / \mathbf{P}_{\mathbf{1 0 0 0 0 \mathrm { BCH }}}[-]$ & - & 1.36 & $\mathbf{P M F} / \mathbf{Q}_{\mathbf{1 0 0 0 0 ~ B C H}}[-]$ & - & 1.38 \\
\hline
\end{tabular}

Afin d'expliciter ces différences, un travail supplémentaire de comparaison des séries de pluies observées utilisées pour chacune des différentes estimations et pour chaque bassin versant est nécessaire. Pour le bassin de la Coquitlam, il apparait en effet que les estimations MEWP dépendent fortement de la série de pluies de bassin considérée et donc des hypothèses de constitution des pluies de bassin : le ratio estimé avec la pluie de bassin EDF (caractérisée par une pluie moyenne annuelle de $4212 \mathrm{~mm}$ ) est inférieur à celui obtenu avec la pluie de bassin $\mathrm{BCH}$ (caractérisée par une pluie moyenne annuelle de $3824 \mathrm{~mm}$ ). Enfin, l'utilisation de la classification de types de temps définie par Brigode et al. [2013] pour la définition de nouvelles valeurs de PMP - par exemple une valeur de PMP par type de temps - permettrait une comparaison d'estimations plus rigoureuse.

\section{IV.2. Estimations des débits extrêmes}

La Figure 3 présente les distributions des débits moyens journaliers simulés par la méthode SCHADEX comparées aux maxima annuels observés et aux estimations de PMF pour les deux bassins versants.
Les valeurs de PMF estimées par BCH sont largement supérieures aux valeurs de débits journaliers de période de retour 10000 ans estimées par la méthode SCHADEX, pour les deux bassins versants étudiés. Un ratio entre la valeur de PMF et la valeur de débit journalier de période de retour 10000 ans estimée par SCHADEX a été calculé pour chaque bassin versant et est reporté dans la partie droite du Tableau 2.

La transformation pluie-débit réalisée au sein de la méthode SCHADEX, grâce au modèle hydrologique MORDOR, semble être caractérisée par un coefficient d'écoulement plus faible par rapport au modèle hydrologique UBC Watershed Model, utilisé lors de l'estimation de la PMF. En effet, à volume de pluies extrêmes précipité et à conditions de saturation des bassins versants comparables (les ratios entre PMF et pluies de période de retour 10000 ans sont proches de 1 et différents états de saturation observés sont considérés dans les deux méthodes), le modèle UBC Watershed Model entraine une estimation de PMF largement supérieure aux débits extrêmes estimés par le modèle MORDOR et la méthode SCHADEX. Enfin, il est intéressant de comparer les ratios entre estimations

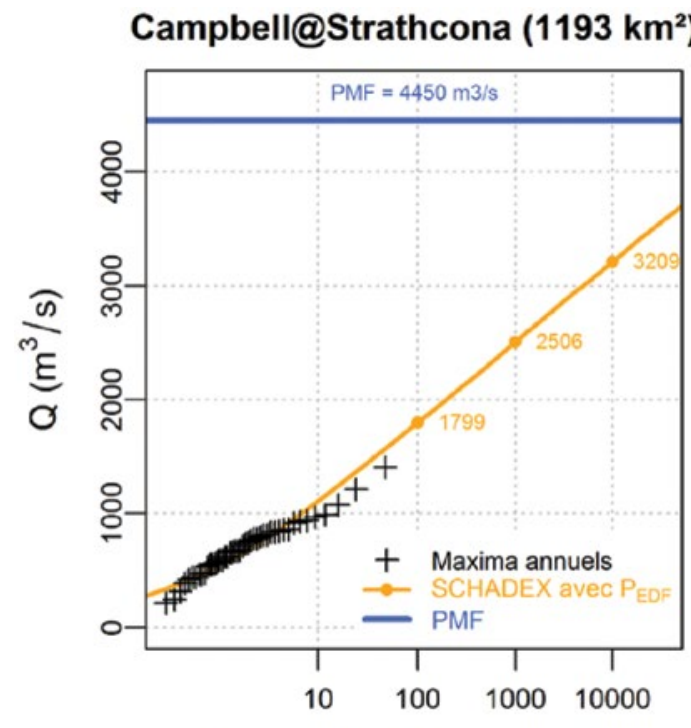

Temps de retour (années)

\section{Coquitlam@Coquitlam (188 km²)}

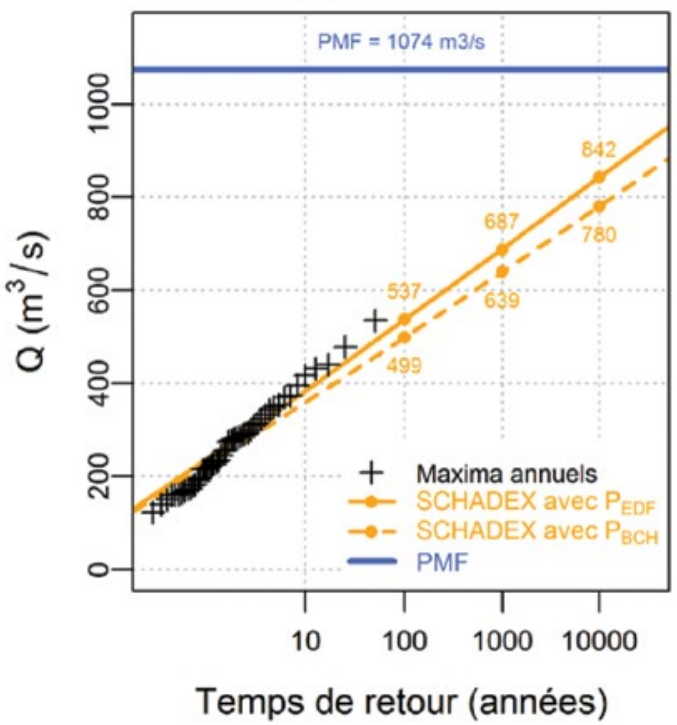

Figure 3 : Bassins versants de la Campbell (à gauche) et de la Coquitlam (à droite) : distributions des débits moyens journaliers simulés par la méthode SCHADEX en utilisant la pluie de bassin construite par EDF (lignes orange) et en utilisant la pluie de bassin construite par BCH (tirets orange), comparées aux distributions de maxima annuels observés (croix noires) et aux estimations de PMF réalisées par BCH (ligne bleue). 
de pluies extrêmes (calculés dans la section précédente) et les ratios entre estimations de débits extrêmes pour chaque bassin, et de noter que ces ratios s'inversent : le ratio de pluies extrêmes le plus faible est calculé pour le bassin de la Campbell (0.94 comparé à 1.11) alors que le ratio de débits extrêmes le plus faible est calculé pour le bassin de la Coquitlam (1.28 comparé à 1.39).

Pour le bassin de la Coquitlam, l'écart entre valeurs extrêmes simulées par la méthode SCHADEX en considérant la pluie de bassin EDF, et en considérant la pluie de bassin $\mathrm{BCH}$ est beaucoup plus important pour les pluies extrêmes que pour les débits extrêmes. Ce résultat s'explique par le calage du modèle hydrologique, qui « dilue » les différences entre les entrées de pluies, puisqu'étant contraint à s'approcher des débits journaliers observés par la fonction objectif utilisée.

\section{CONCLUSIONS}

Deux méthodes d'estimations des débits extrêmes ont été appliquées sur deux bassins versants (le bassin versant de la Campbell au barrage de Strathcona $\left(1193 \mathrm{~km}^{2}\right)$ et le bassin versant de la Coquitlam au barrage de la Coquitlam (188 $\left.\mathrm{km}^{2}\right)$ ) de Colombie-Britannique : la méthode déterministe PMP/PMF et la méthode probabiliste SCHADEX.

Les valeurs de PMP estimées par BCH ont tout d'abord été comparées avec les distributions de pluies journalières obtenues avec le modèle probabiliste de pluie MEWP utilisé dans la méthode SCHADEX. La PMP est inférieure à la valeur de pluie journalière de période de retour 10000 ans estimée par le modèle MEWP pour le bassin de la Campbell. Pour le bassin de la Coquitlam, la PMP est supérieure aux valeurs de pluies journalières de période de retour 10000 ans estimées en considérant les deux pluies de bassin disponibles. Cette comparaison de valeurs extrêmes estimées est incomplète sans un travail supplémentaire de comparaison des séries de pluies observées considérées lors de l'application des différentes méthodes. La considération de deux séries de pluies de bassin différentes (pour le bassin de la Coquitlam) révèle en effet que les estimations MEWP dépendent fortement de la série de pluies de bassin considérée, et donc des hypothèses de constitution des pluies de bassin.

Pour les deux bassins versants de Colombie-Britannique étudiés, les valeurs de PMF sont largement supérieures aux estimations de débits moyens journaliers estimés par la méthode SCHADEX. En effet, les ratios entre les valeurs de PMF et les débits journaliers moyens de période de retour 10000 ans sont compris entre 1.28 et 1.39 . Ces valeurs de ratios sont du même ordre de grandeur que celles obtenus lors de la comparaison de valeurs de PMF et d'estimations de débits extrêmes réalisées avec la méthode du GRADEX [Guillot et Duband 1967] pour 43 bassins versants différents; pour lesquels les valeurs de ratios étaient comprises entre 1.08 et 3.52 [CFGB, 1994]. La Figure 4 permet de comparer ces différentes valeurs de ratios en fonction de la taille des bassins versants.

La transformation pluie-débit réalisée par le modèle hydrologique MORDOR au sein de la méthode SCHADEX produit, à conditions de simulations similaires, des estimations de débits extrêmes inférieures à celles estimées grâce à la transformation pluie-débit du modèle UBC Watershed Model au sein de la méthode SEFM et PMF. Une comparaison des structures et des performances des deux modèles forcés par les mêmes entrées pourrait expliciter ces résultats. De plus, une perspective intéressante pourrait être de substituer le modèle hydrologique MORDOR (classiquement utilisé dans la méthode SCHADEX) par le modèle hydrologique UBC Watershed Model.

Cette communication illustre la grande nécessité de réaliser des estimations de pluies et crues extrêmes avec les mêmes entrées hydroclimatiques (et notamment des séries de pluies de bassin construites avec les mêmes hypothèses) pour réaliser une comparaison rigoureuse de méthodes de prédétermination.

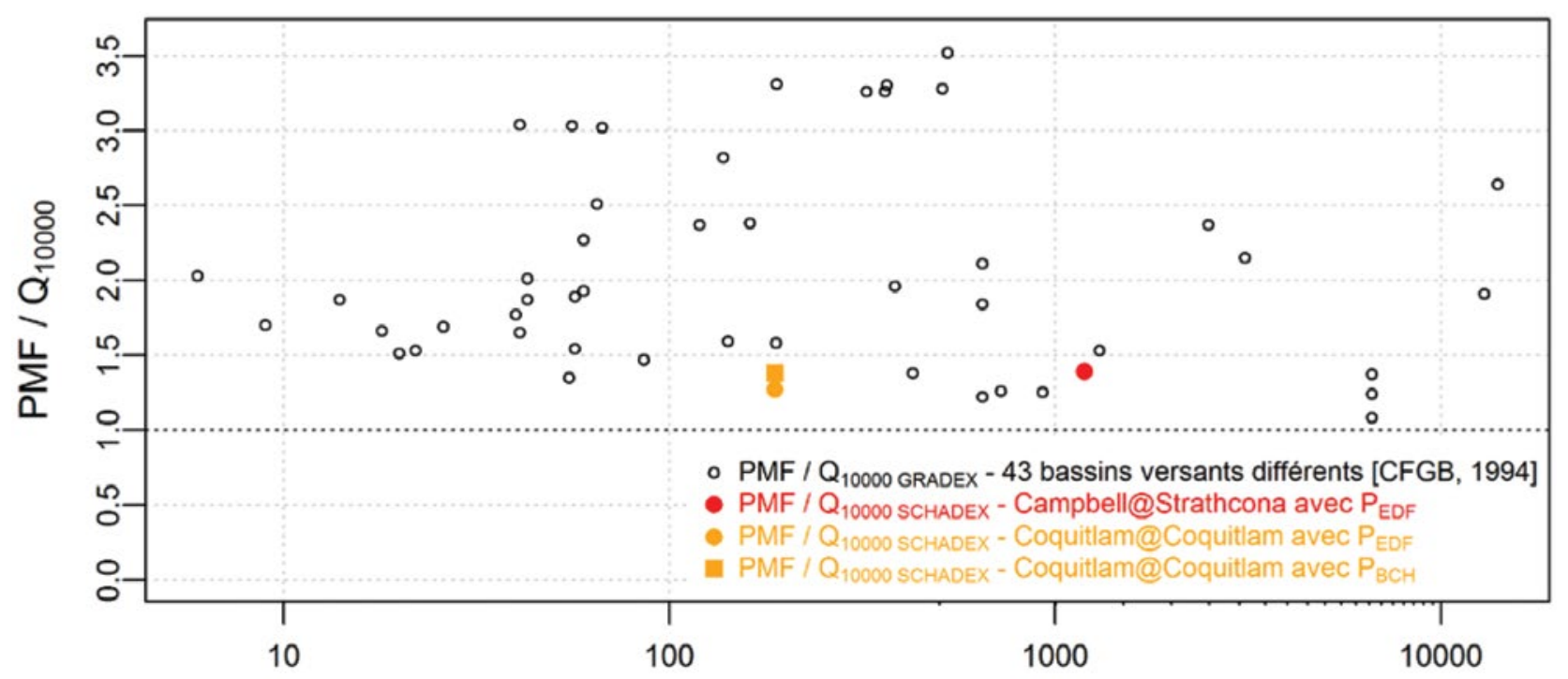

Aire de bassin versant $\left(\mathrm{km}^{2}\right)$

Figure 4 : Ratios calculés entre des valeurs de PMF et de débits de période de retour 10000 ans (estimés par les méthodes GRADEX (points blancs) et SCHADEX (points et rectangles colorés)) en fonction des aires de bassins versants considérés. 


\section{RÉFÉRENCES}

Brigode P., Mićović Z., Bernardara P., Paquet E., Garavaglia F., Gailhard J. Et Ribstein P. (2013) - Linking ENSO and Heavy Rainfall Events over Coastal British Columbia Through a Weather Pattern Classification. Hydrology and Earth System Sciences. 17 (4) : 1455-1473, doi 10.5194/hess-17-1455-2013

CDA (2007) - Canadian Dam Association Dam Safety Guidelines. 88 pages

CFGB (1994) - Les crues de projet des barrages: méthode du GRADEX. 18ème congrès CIGB-ICOLD, Durban, South Africa, 1994. Bulletin du Comité Français des Grands Barrages

Garavaglia F., Gailhard J., Paquet E., Lang M., Garçon R. Et Bernardara P. (2010) - Introducing a Rainfall Compound Distribution Model Based on Weather Patterns Sub-sampling. Hydrology and Earth System Sciences. 14 (6) : 951-964, doi 10.5194/hess-14-951-2010

Garavaglia F., Lang M., Paquet E., Gailhard J., Garçon R. Et RENARD B. (2011) - Reliability and Robustness of Rainfall Compound Distribution Model Based on Weather Pattern Sub-sampling. Hydrology and Earth System Sciences. 15 (2) : 519-532, doi 10.5194/hess-15-519-2011

Garavaglia F. (2011) - Méthode SCHADEX de prédétermination des crues extrêmes. Méthodologie, applications Études de sensibilité. Thèse, Université de Grenoble. http://hal. archives-ouvertes.fr/tel-00579507/

GARÇON R. (1999) - Modèle global Pluie-Débit pour la prévision et la prédétermination des crues. La Houille Blanche. (7-8) : 88-95, doi 10.1051/lhb/1999088
Guillot P. Et Duband D. (1967) — La Méthode du Gradex pour le calcul de la probabilité des crues à partir des pluies. $I A S H$ Publication. 84

HAnsen E. M. (1987) - Probable Maximum Precipitation for Design Floods in the United States. Journal of Hydrology. 96 (1-4) : 267-278, doi 10.1016/0022-1694(87)90158-2

Mićović Z. Et Quick M.C. (1999) - A Rainfall and Snowmelt Runoff Modelling Approach to Flow Estimation at Ungauged Sites in British Columbia. Journal of Hydrology. 226 (1-2) : 101-120, doi 10.1016/S0022-1694(99)00172-9

Mićović Z., Schaefer M.G. Et TAYlor G.H. (2012) - A Methodology for Analysis of Uncertainty in Probable Maximum Precipitation. 24ème congrès CIGB-ICOLD, Kyoto, Japan

Paquet E., Gailhard J. Et Garçon (2006) - Evolution de la méthode du Gradex : approche par type de temps et modélisation hydrologique. La Houille Blanche. 5 : 80-90, doi 10.1051/ lhb:2006091

Paquet E., Garavaglia F., Gailhard J. Et Garçon (2013) The SCHADEX Method: A Semi-continuous Rainfall-runoff Simulation for Extreme Flood Estimation. Journal of Hydrology. 495 : 23-37. doi: 10.1016/j.jhydrol.2013.04.045

Quick M. C. (1995) — The UBC Watershed Model. Computer Models of Watershed Hydrology (ed. par V. J. Singh). Water Resources Publications, P.O. Box 260026, Highlands Ranch, Colorado, USA

Schaefer M.G. Et BARKer B.L. (2009) - Stochastic Event Flood Model - User Manual. MGS Engineering Consultants Inc., January 